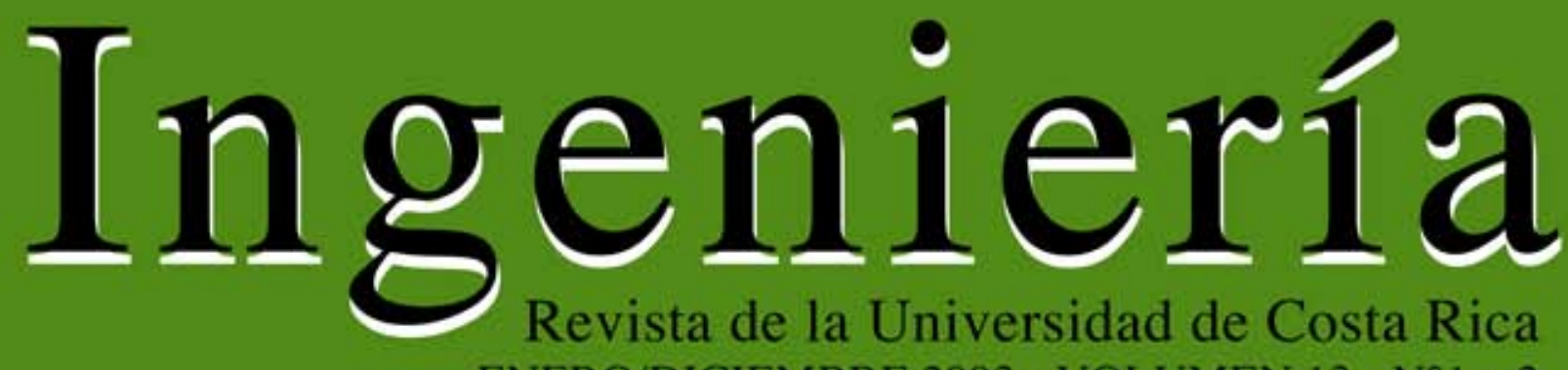
ENERO/DICIEMBRE 2003 - VOLUMEN 13 - N¹ y 2

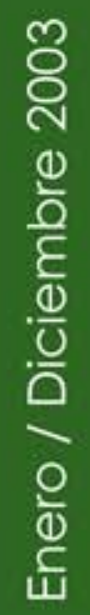

$\frac{m}{5}$
$\frac{c}{d}$
$\frac{5}{5}$
$\frac{\partial}{\circ}$

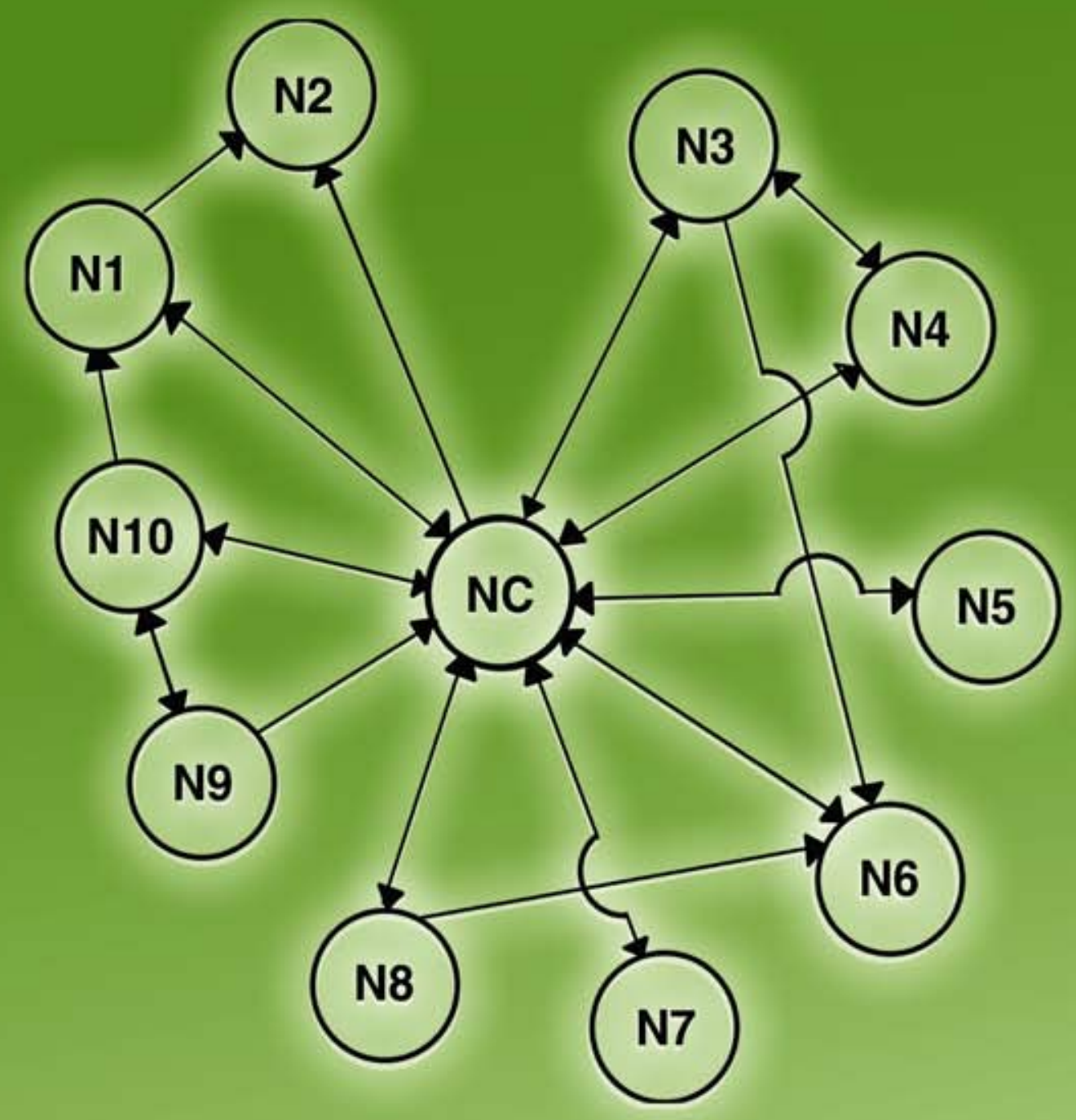

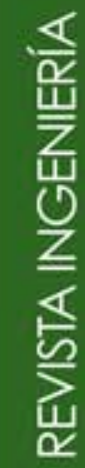




\title{
SISTEMA NACIONAL DE INFORMACIÓN EN AMBIENTE Y SALUD (SINFASCOR)
}

Oscar Coronado Jurado

\begin{abstract}
Resumen
El artículo explica la primera fase de desarrollo del Sistema Nacional de Información en Ambiente y Salud, financiado por OPS y teniendo como contraparte el Ministerio de Salud de Costa Rica. Se usa las teorías de redes recurrentes y los más recientes métodos de diagramación del análisis de sistemas.
\end{abstract}

Palabras clave: Sistemas de información, teoría de redes, ambiente y salud, contenidos de trasiego, nodo, enlace, red, teleinformática, diagrama lógico, diagrama físico, diagrama de contexto.

\begin{abstract}
This article explains the first phase of development of the National Information System of environment and health, financed by OPS and with the counter part Costarican Ministry of Health. It uses the network theory as a recurrent way and the most recent diagram methods of system analysis.
\end{abstract}

Keywords: Information systems, networks theory environment and health, in transit contents, node, link, network, telematics, logic diagram, physical diagram, context diagram.

\section{INTRODUCCIÓN}

Este documento contiene una sinopsis del trabajo que se realizó en Agosto del 2000 con una cobertura de 12 instituciones que se visualizan como nodos del sistema, concebido como red, llamado Sistema Nacional de Información en Ambiente y Salud (SINFASCOR). Se abarcaron en total 12 instituciones (nodos) y 13 departamentos o entidades de todos ellos con referencias indirectas al menos 25 instituciones más.

Un sistema nacional o regional de información requiere de desarrollos permanentes. Nuestra recomendación es continuar integrando otros nodos a tiempo de que se desarrollen los nodos actuales con el concepto de cliente / proveedor; como de Conjuntos o Contenidos de Trasiego que consisten en indicadores, datos, información gráfica o combinación de éstos, dotando de equipo y con los desarrollos locales (nodo - institución), de enlaces (medios de transmisión), software y la red inicial en su conjunto, incluyendo peajes (por información que se vende) y dispositivos de seguridad.

El artículo en primer lugar presenta un diagrama físico que integra las bases, los temas abordados en ambiente y salud, los nodos, sus enlaces, sus usuarios proveedores y los sectores sociales, productivos y de servicios, actores gubernamentales y de la sociedad civil, y en general los beneficiarios reales y potenciales del sistema. 
Posteriormente se presenta un diagrama nivel cero que ilustra al acceso a SINFASCOR.

Por último se presenta para cada uno de los nodos un diagrama de contexto (Nivel 1), que muestra su relación con la red inicial y los flujos reales o potenciales con un conjunto de otros organismos, muchos de los cuales no fueron estudiados directamente en esta I Fase del desarrollo de SINFASCOR.

Para cada nodo además se detallan diagramas de flujos de datos lógicos tanto de nivel cero como de nivel 1 ( los de nivel cero son generales, los de niveles 1 más detallados y especificados), que muestran las entidades, los sistemas de información implicados, documentos, software y productos, es decir salidas del sistema y su relación con el nodo central de SINFASCOR

\section{ALGUNOS ASPECTOS METODOLÓGICOS}

Aparte de la diagramación normalizada, se utilizó un enfoque de redes, adaptado por el autor y explicado en (Coronado, 2003) A riesgo de sobresimplificar, el enfoque consistió en elegir una institución nodo, de alto nivel de desarrollo en el tema, el Observatorio del Desarrollo de la Universidad de Costa Rica, y seleccionar un conjunto de indicadores, sistemas de información, base de datos, Sistemas de Información Geográfica, y utilizarlos como una base para precisar que requería y podía producir otra institución-nodo. Esto hasta llegar a 12 instituciones, pero en forma interactiva, porque con determinados resultados (requerimientos/ productos) se haría necesario volver sobre la secuencia de nodos, hasta completar varias interacciones sobre los doce, de forma que se tuviera núcleo preciso de requerimientos, y los productos reales y potenciales de conjuntos de indicadores; sistemas de información, bases de datos, sistemas de información geográficos, que llamaremos sitemas de trasiego.

No todos los nodos están conectados entre sí, salvo con el nodo central; sólo en los casos en que las transacciones son del tipo productor/ usuario; es decir, que ambas instituciones reciben y suministran datos entre sí.

\section{Nodo central:}

Administrador de sistema.

\section{Nodo productor:}

Produce información / datos Primarios o Secundarios. El productor Primario produce datos / información en la propia institución por trabajo de campo y otros datos. El Secundario usa datos de otras fuentes y los procesa suministrando nuevos datos.

\section{Nodo usuario:}

Usa información / datos de otros nodos, no suministra.

\section{Nodo Productor / usuario:}

Produce datos / información y recibe de otros nodos, como ejemplo vease la Figura 1.

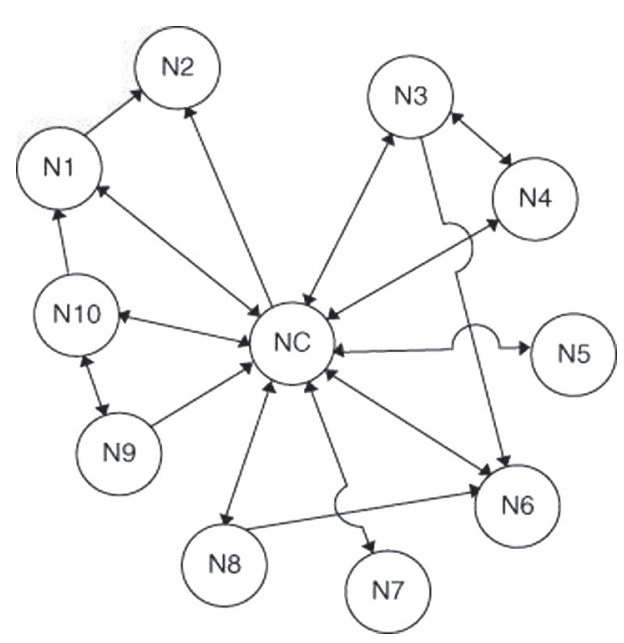

Figura 1. Enfoque de redes 
En la Figura 2 N2 es un modo usuario, N10 y N9 son productor / usuario entre sí, N1 en su enlace con $\mathrm{N} 10$ es usuario, pero con N2 es productor.

En síntesis, este enfoque permite visualizar la ubicación relativa de cada institución en el sistema, y visualizar la naturaleza de las transacción, tanto de volumen como de periocidad de datos e información para especificar el equipamiento informático y de telecomunicaciones requerido.

Esto es apenas una clasificación; el uso de nodos como concepto implica la posibilidad de otras categorizaciones más complejas. Por lo pronto y para efectos de esta fase de SINFASCOR, el enfoque de redes permite visualizar la ubicación de cada institución en el sistema, visibilizar la naturaleza de las transacciones, tanto en volumen como en periocidad de datos e información, para especificar el equipamiento teleinformático requerido. También permite planificar el desarrollo de proveedores asignando los recursos para ello.

Se hace una descripción codificando cada flujo (Conjunto) en términos de requerimientos reales y potenciales, y productos reales de cada nodo, con diferentes niveles de precisión y detalle en dependencia de la claridad y precisión de los entrevistados, de sus requerimientos y sus criterios de experto y de la posibilidad de otras instituciones de suplirlos y también del desarrollo desigual de los nodos - instituciones - departamentos.

\section{ACERCAMIENTO INICIAL A SINFASCOR}

A continuación se presenta un acercamiento a la profundidad y cobertura del estudio en las instituciones abarcadas en forma resumida:

\section{Observatorio de Desarrollo (OdD - UCR)}

- Requerimientos Ambientales / Salud (instrumento).

- Inventario Teleinformático.

\section{Producto Principal:}

- CD ROM: Tendencias del Desarrollo en CR con más de 1000 indicadores de salud, ambiente, calidad de vida.

- Boletines Informativos sobre biodiversidad, administración, corrupción, entre otros.

2. Instituto Nacional de Vivienda y Urbanismo (INVU)

\section{(División de Planificación Territorial)}

- Requerimientos Ambientales / Salud (instrumento)

- Inventario Teleinformático.

\section{Comisión Nacional de Emergencias} (CNE)

- Requerimientos Ambientales / Salud (instrumento).

- Base de Datos de recursos disponibles en caso de desastre (número de escuelas, gimnasios, ubicación de planteles del MOPT, etc).

\section{Productos y Centros Principales:}

- Sistema de Información Integrado para Emergencias.

- Información de Áreas de Amenaza Geológica, Tecnológica e Hidrometrológica.

- Red Nacional de Comunicaciones (incluye ministerios, laboratorios de sismología y vulcanología, policía, tránsito, Cruz Roja, CCSS, entre otros).

- Inventario Teleinformático y de radiocomunicación. 
4. Instituto Costarricense de Acueductos y Alcantarillados (ICAA)

\subsection{Departamento de Estudios Básicos}

Productos Principales:

- $\quad$ Base de Datos de Aforos.

\subsection{Sistema de Información del ICAA}

Productos Principales:

- $\quad$ Base de Datos de la GAM (Gran Área Metropolitana).

- Mapas Digitales de toda la GAM.

\subsection{Dirección Aguas Subterráneas}

Productos Principales:

- Base de Datos de Aforos.

- Inventario Teleinformático.

\subsection{Departamento de Cuencas}

- Requerimientos Ambientales / Salud.

- Inventario Teleinformático.

\subsection{Departamento de Estudios y Proyectos}

- Requerimientos Ambientales.

- Inventario Teleinformático.

\section{Servicio Nacional de Riego y} Avenamiento (SENARA)

\section{(Departamento de Aguas Subterráneas)}

Productos Principales:

- $\quad$ Base de Datos de Aforos.
- Requerimientos Ambientales / Salud (Instrumento).

- Inventario Teleinformático.

\section{Municipalidad de Belén}

\subsection{Unidad Ambiental}

- Requerimientos Ambientales (Instrumento).

- Inventario Teleinformático.

\section{Red de información del Sector} Agropecuario (INFOAGRO)

Productos y Centros Principales:

- Centros Rurales de Información: facilitan información en la cadena agroproductiva.

- Página Web con información de cultivos / tecnología / mercado / comercio internacional / información geográfica / proyectos agropecuarios.

8. Laboratorio de Química de la Atmósfera Universidad Nacional (UNA)

- Requerimientos Ambientales (Instrumento).

Productos Pincipales:

- Información de presencia de metales en el aire.

- Información sobre emanaciones volcánicas (Volcán Poás).

- Prestación de Servicio a Industrias sobre análisis de emanaciones.

- Inventario Teleinformático. 
9. Ministerio de Obras Públicas y

Transportes (MOPT)

\subsection{Instituto Geográfico Nacional}

- Requerimientos Ambientales (Instrumento).

Productos Principales:

- Fotos aéreas de de Costa Rica.

- Mapas:

- Físicos.

- Políticos.

-Uso de la tierra.

- Curvas de nivel.

- Pendientes.

- Geológico.

- Inventario de equipo de cómputo.

10. Ministerio de Ambiente y Energía (MINAE)

\subsection{Sistema Nacional de Áreas de} Conservación

Productos Principales

Información de:

- Áreas Protegidas.

- Humedales.

- Reserva Biológica.

- $\quad$ Parque Nacional.

- Monumento Nacional.

- Refugios de Vida Silvestre.

- Fincas Propiedad del Estado.
- Zonas Protectoras

- Área Boscosa.

- Requerimientos Ambientales / Salud (Instrumento).

- Inventario Teleinformático.

11. Caja Costarricense del Seguro Social

11.1 Departamento de Saneamiento Básico y Ambiental Institucional / Salud Ocupacional

Productos Principales:

Información de:

- Cantidad de desechos hospitalarios.

- Tipo de infecciones por hospital y región.

- Requerimientos Ambientales / Salud / (Instrumento).

- Inventario Teleinformático.

11.2 Departamento de Estadística de Servicios de Salud

Productos Principales:

Información de:

- Estadísticas en general de hospitales (número de consultas, horas por operación, consumo de medicamentos, número de placas, etc).

- Requerimientos Ambientales / Salud (Instrumento).

- Inventario Teleinformático. 
12. Ministerio de Salud

\subsection{Departamento de Vigilancia de la Salud}

Productos Principales:

- $\quad$ Estadísticas estratégicas de enfermedades que si fuesen reveladas al público generarían pánico.

- Requerimientos Salud

- Inventario Teleinformático

\subsection{NetSalud}

\section{Productos Principales:}

- Llevan a cabo la publicación de 85 páginas Web.

- Inventario Teleinformático

\subsection{Departamento de Protección al Ambiente Humano}

- Requerimientos Ambientales / Salud (Instrumento).

\subsection{Dirección de Registros y Controles del Ministerio de Salud}

\section{Principales Productos:}

Bases de Datos de:

- Medicamentos y Cosméticos.

- Precursores.

- Alimentos.

- Químicos.

- Materia Prima.
- Trámites.

- Establecimientos.

- Inventario Teleinformático.

- SIMARDE (En una etapa posterior se abordará SISVICA).

\section{DIAGRAMAS Y NIVELES DE ACCESIBILIDAD - SEGURIDAD PARA EL SINFASCOR}

Se levantaron los siguientes diagramas tal y como se ha explicado:

- Diagrama Físico del SINFASCOR: explicando las conexiones del sistema entre las diferentes entidades, diseñado de tal manera que son visibles los servidores, tipos de conexiones y diferentes recursos informáticos y documentales con que cuenta cada institución (ver Figura 2).

- Diagrama Lógico del SINFASCOR: haciendo referencia a las diferentes bases de datos que componen el sistema y su interrelación (no se presenta por razones de espacio).

- Diagrama Nivel Cero de Acceso al SINFASCOR: en este diagrama se pone de manifiesto cómo un usuario desde su terminal entra al sistema, se le permite acceder a cierto tipo de información, realiza su consulta y finalmente emite el reporte (ver Figura 3).

- Diagramas de Contexto por institución en el SINFASCOR con los respectivos flujos de datos: explican las conexiones de información entre usuarios y proveedores del sistema, es decir, de cual proveedor depende cada usuario (ver Anexo 1). 
- Clasificación de Indicadores en Tierra / Agua / Aire / Sonido / Aspectos Climáticos / Salud: para homogeneizar los requerimientos de las diferentes instituciones del sistema, se clasificó la diferente información en 6 grandes temas, con el fin de que se visualice de una manera más clara el tipo de formación que enlaza a cada usuario y proveedor.
- Seguridad y Software del Sistema: para finalizar se presentan las recomendaciones de desarrollo e instalación de software en cada nodo y el nodo central, y así concluir con la dotación de infraestructura y equipamiento informático, de seguridad, peajes y conectividad. No se incluye construcción civil. 


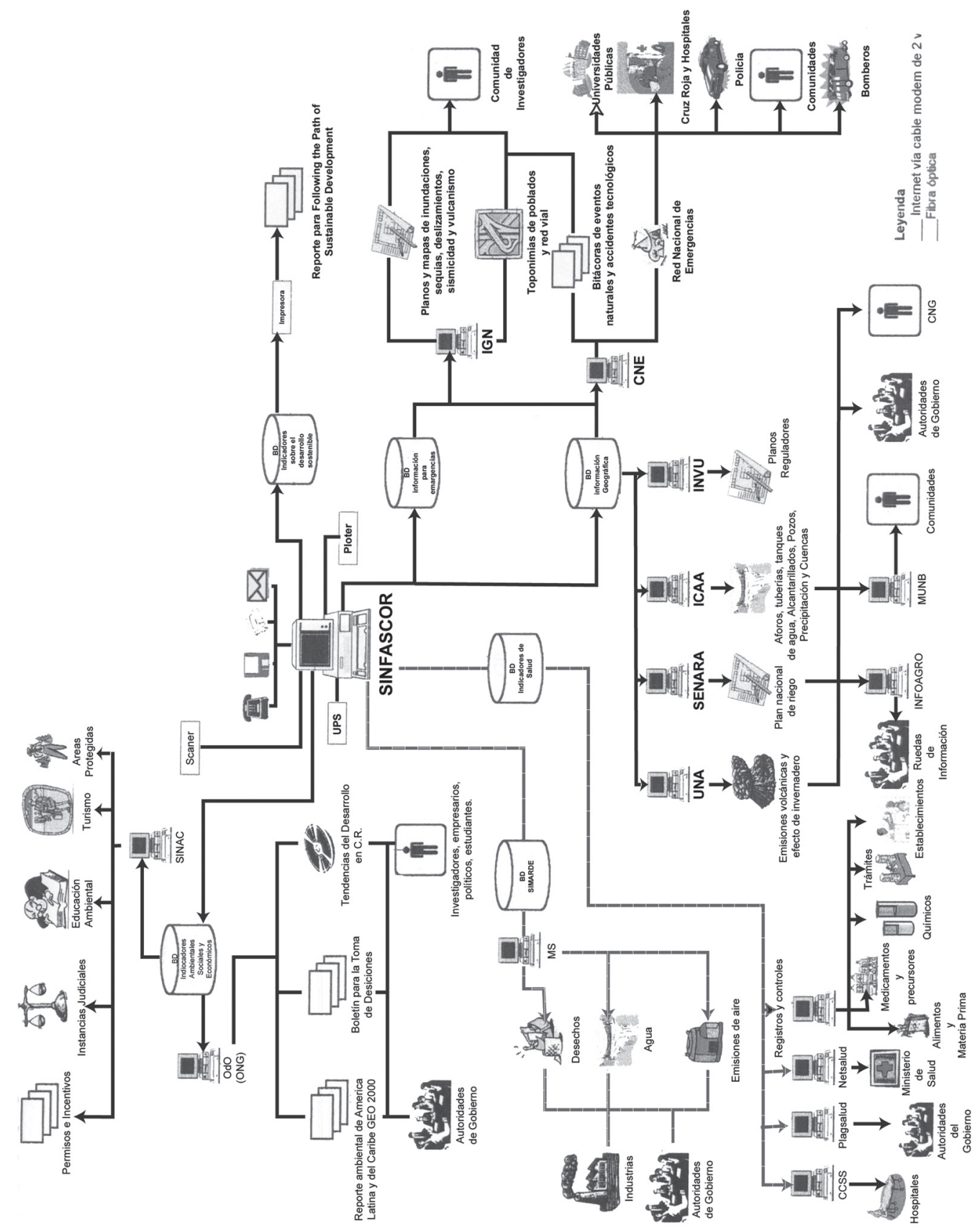

Figura 2. 

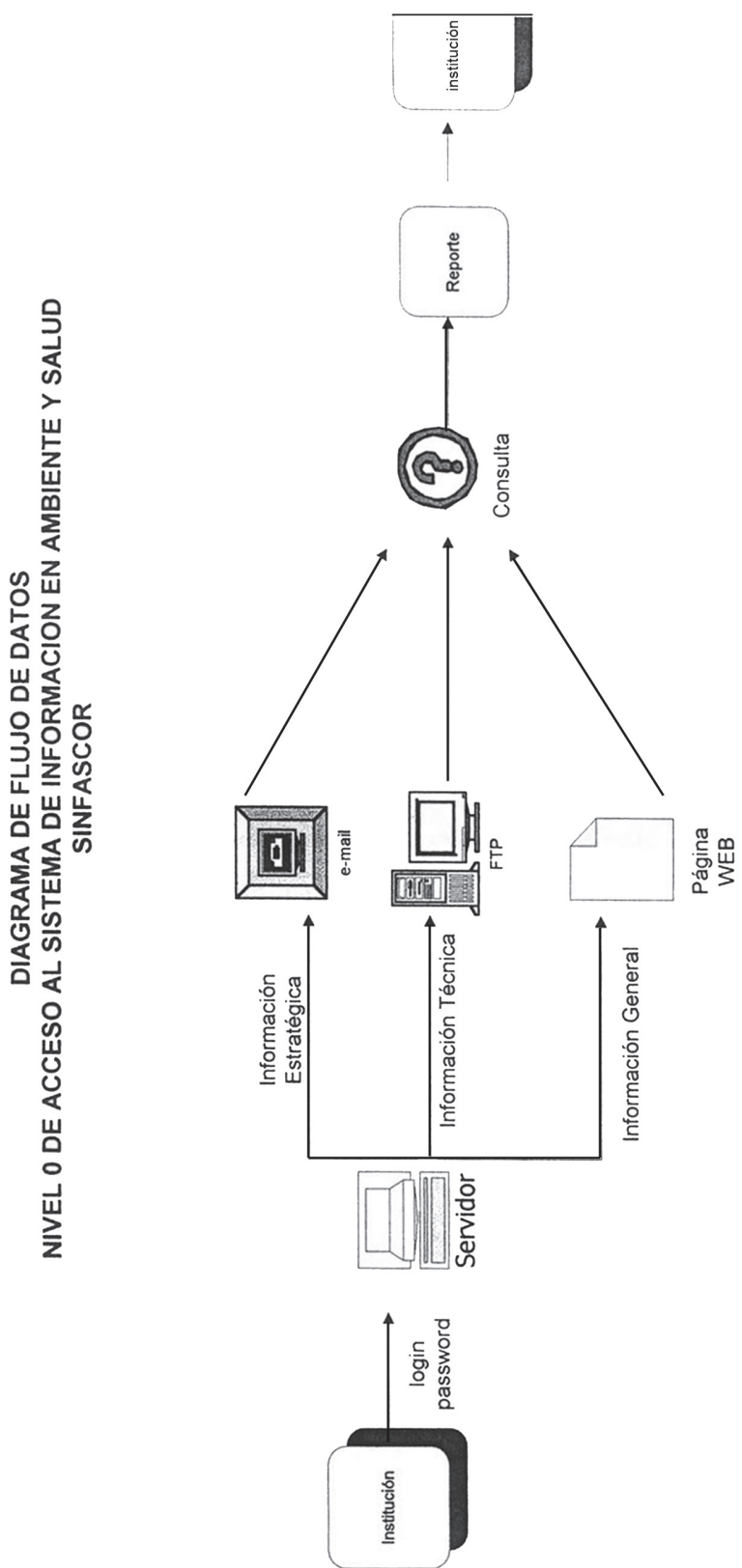

Figura 3. 


\section{IMPORTANCIA DE SINFASCOR}

El Sistema de Información en Ambiente y Salud de Costa Rica es una iniciativa que tanto el Gobierno de la República, como organismos de la sociedad civil y la Organización Panamericana de la Salud, se han planteado para el logro de una instancia interinstitucional que ligue las instituciones públicas y privadas del país, en un conjunto sistematizado de usuarios / proveedores, con claras reglas de actuación y con especificación clara de competencias. Esto con una base computacional centralizada para la administración del recurso informático en ambiente y salud, eliminando duplicidades, desarrollando proveedores y usuarios en el marco de un contrato interorganizacional sostenible.

Este, sería el primer sistema de información nacional de Costa Rica, que merezca tal denominación, en virtud de la calidad de sus productos, en un contexto de cooperación que asegure la protección de información que es vendida por algunos organismos a sectores con intereses privados, no así a organismos sin fines de lucro.

El objetivo del proyecto fue determinar las especificaciones a nivel lógico y físico, que darán cabida a las posteriores etapas de diseño de bases de datos, especificaciones de control y seguridad, programación, pruebas y conversión - capacitación.

El trabajo se realizó en un periodo de 3 meses con el apoyo de una solicituddel Ministerio de Salud a un mínimo de 30 organismos.

Aún con la nota del ministro, fue necesario acudir en su gran mayoría, a contactos informales que, a su vez, enlazaran al grupo consultor con expertos, jefaturas de dependencia y a un amplio acerbo bibliográfico que permitió dirigir el trabajo de forma que se lograra la más amplia cobertura y rigurosidad. La concepción del sistema, como se ha explicado, es la de una red que se llevó a configurar con 12 instituciones (nodos) con la concepción de nodo como proveedor, como cliente o como combinación de éstos, es decir el establecimiento lógico de los enlaces internodos, para después identificar los medios de transmisión.

Aparte de los diagramas descritos en el apartado 4 se hace, una descripción codificando cada flujo en términos de requerimientos reales y potenciales y productos reales de cada nodo, con diferentes niveles de precisión y detalle, en dependencia de la claridad de los entrevistados, de sus requerimientos y sus criterios de experto; de la posibilidad de otras instituciones de suplirles y también del desarrollo desigual de los nodos.

Para concluir, se presentan las recomendaciones de desarrollo e instalación de software $\mathrm{y}$ aplicaciones en cada nodo, y en el nodo central. Esto con el objetivo de finalizar con la especificación de infraestructura y equipamiento informático de seguridad, peajes y conectividad.

Obviamente, el proceso es recursivo e implica en fases posteriores, integrar nuevos nodos y consultar nuevos requerimientos y potencial de suministrar conjuntos de información de nodos ya abordados en este estudio.

El desarrollo de sistemas de información, a escala organizacional, en Costa Rica en el sector público (y en gran medida del sector privado) ha estado caracterizado por grandes distorsiones, producto de muchos factores que van desde la cultura organizacional, el estilo de desarrollo de los sistemas de información y, en general, de asimilación de tecnologías de información hasta los enfoques reduccionistas de profesionales y técnicos en la materia y los intereses de los proveedores.

\section{CONCLUSIONES}

Es importante enfatizar que además de la concepción subyacente del sistema como red, a su vez se utilizaron redes informales de contactos. 
El resultado permitió acumular y sistematizar un gran volumen de especificaciones de conjuntos, datos / información, así como multiplicidad de interrelaciones.

Es también importante que se entienda que a lo largo del trabajo, se tendrán multiplicados los conjuntos: indicador, datos, información gráfica o combinación de ellos, en virtud de que hay nodos que son ya sea productores primarios o secundarios de estos conjuntos y son usuarios reales o potenciales de ellos. Esto es una realidad consecuencia del estado desigual de desarrollo de nodos-enlaces y sistemas de información como compartimentos-estancos, con una cultura prevalente de feudo y contactos bilaterales de trasiego de información; basados en el contacto personal con escaso acuerdo interinstitucional.

Durante el desarrollo del SINFASOR, se pudo evidenciar que la componente que se podría denominar cultural, es uno de los principales obstáculos para el desarrollo de sistemas de información interinstitucionales.

En apretada síntesis, se planteará que esta experiencia visibiliza que elementos de esa cultura al menos en el sector público, se reproducen y tienden a consolidarse.

El problema del poder que se le asigna a la posición de información, deriva en el desarrollo de aplicaciones locales en una misma organización, que manejan bajo formatos diferentes en todo o en parte la misma información o datos. Esto es más arraigado a nivel interinstitucional. El otro elemento de la cultura de poder, lo constituye la adquisición de hardware y software como símbolos de status y posicionamiento en la organización. Esto frecuentemente no se deriva en altos réditos a nivel de productividad en la automatización de procesos organizacionales, ni la mejoría en la toma de decisiones.

En ese extremo predomina la subutilización endémica de la capacidad informática.

En el otro extremo ocurre lo contrario: dependencias de gran potencial y necesidad de recursos informáticos tienen un equipamiento caduco y aplicaciones deficientes. Además pese a todas las facilidades que la tecnología ofrece; la opción por plataformas cerradas, y la ausencia de esquemas mínimos de planeamiento, deriva en que el problema de la compatibilidad prevalece.

En suma si se hubiera planteado lo siguiente como hipótesis en el desarrollo de SINFASCOR; se podría explicar la ausencia en Costa Rica de verdaderos sistemas de información nacionales.

La cultura o infracultura de los compartimentosestancos, de la adquisición caótica de equipos y software, la necesidad de no depender de otros organismos (autarquía) y la concepción de información como fuente de poder, ha provocado un estado entrópico de los sistemas de información institucionales. A escala interinstitucional, los contratos generalmente de palabra de intercambio de información bilateralmente, en condiciones de informalilidad, sin aseguramiento de la calidad ni la oportunidad en el suministro de ese tipo de bienes son la constante. Es así, como hay grandes vacíos en muchas áreas y por otro lado, unas cuantas instituciones que producen parcelas idénticas de algún componente de información. 


\section{Anexo 1}

\section{DIAGRAMAS DE CONTEXTO}

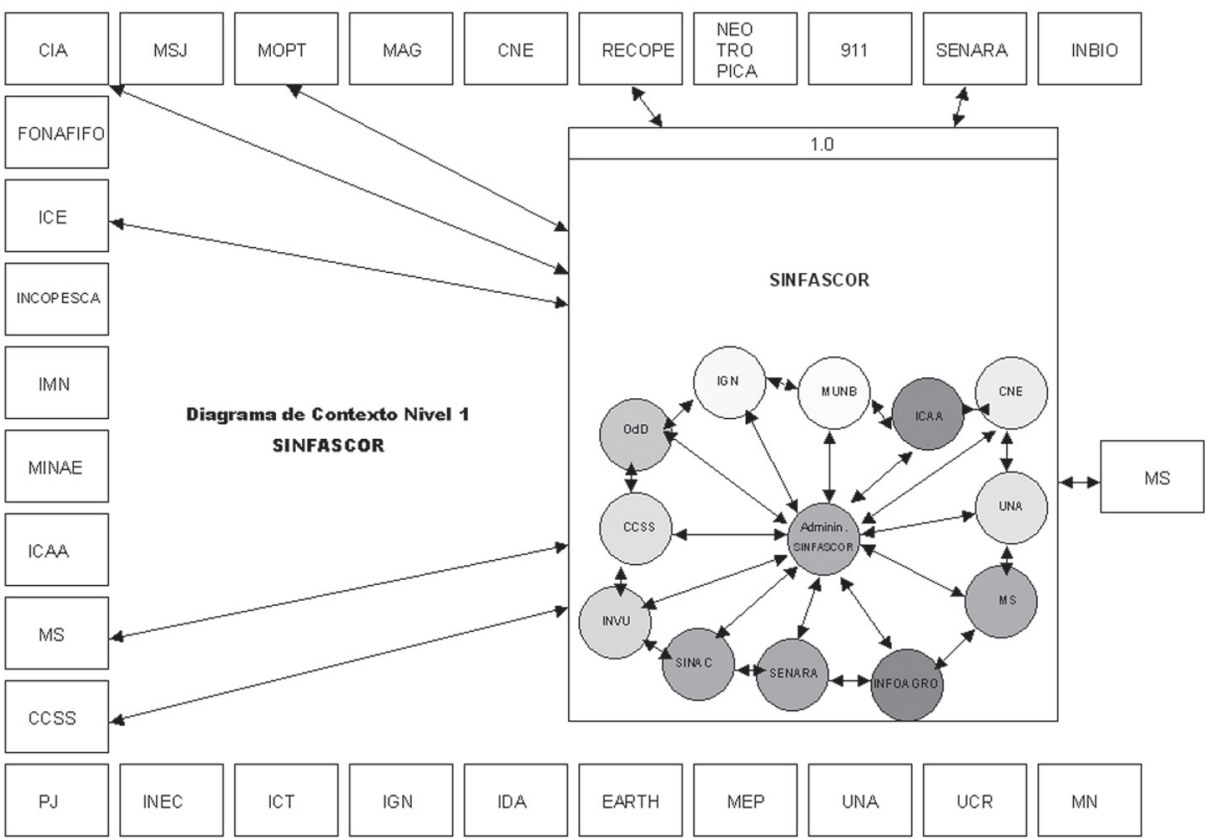

Figura A.1

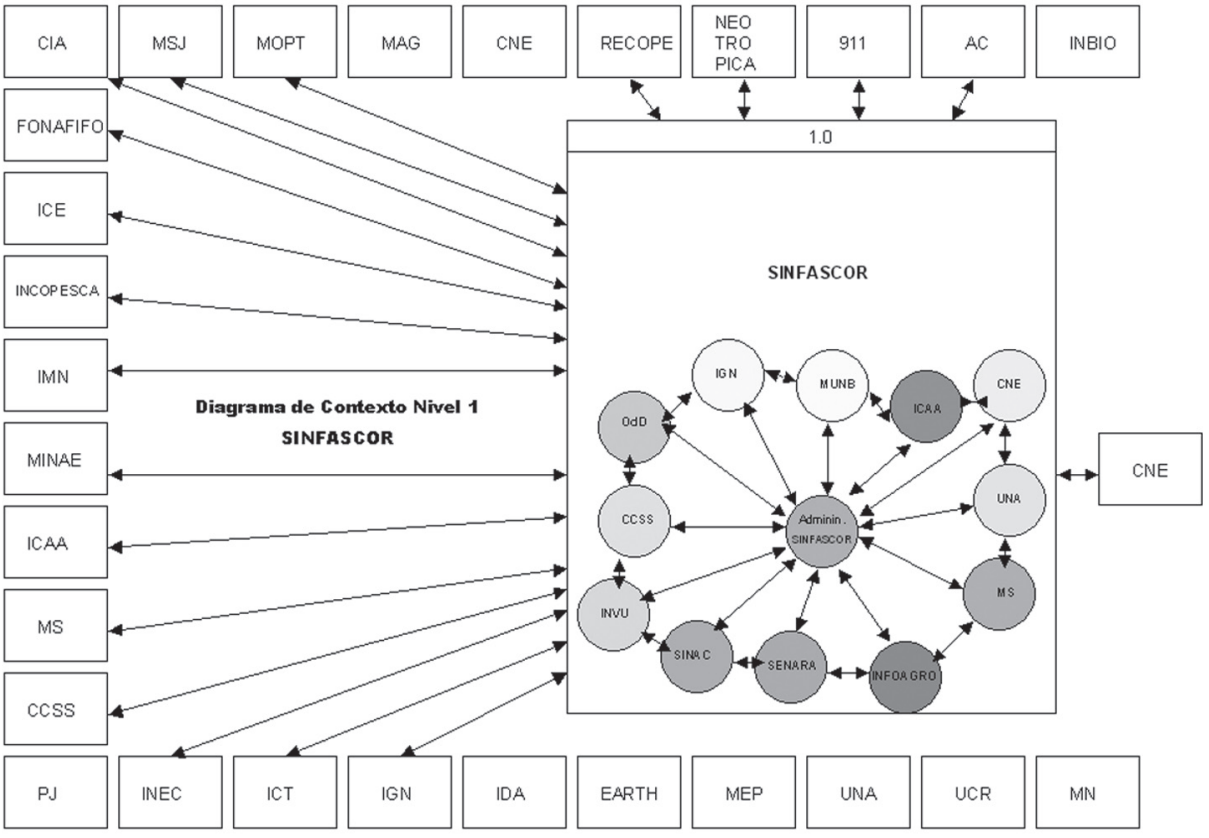

Figura A.2 


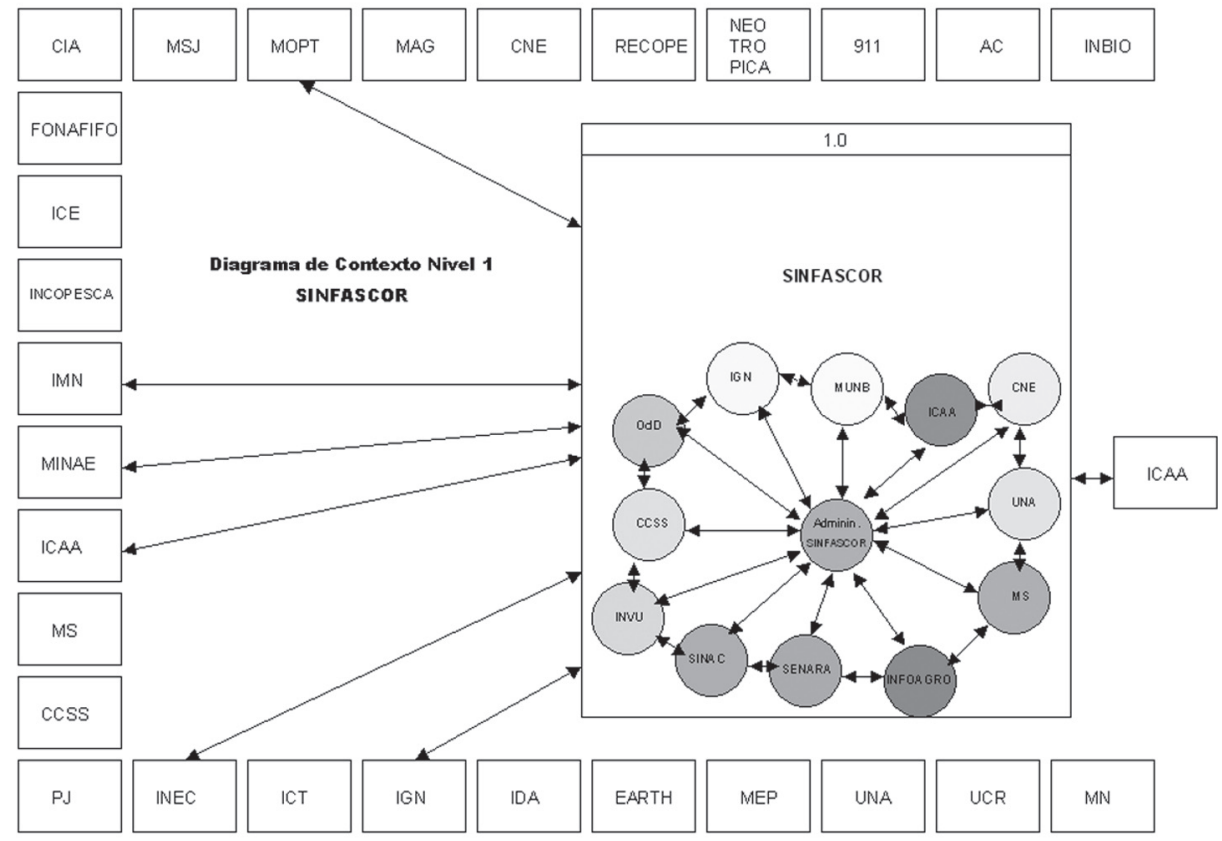

Figura A.3

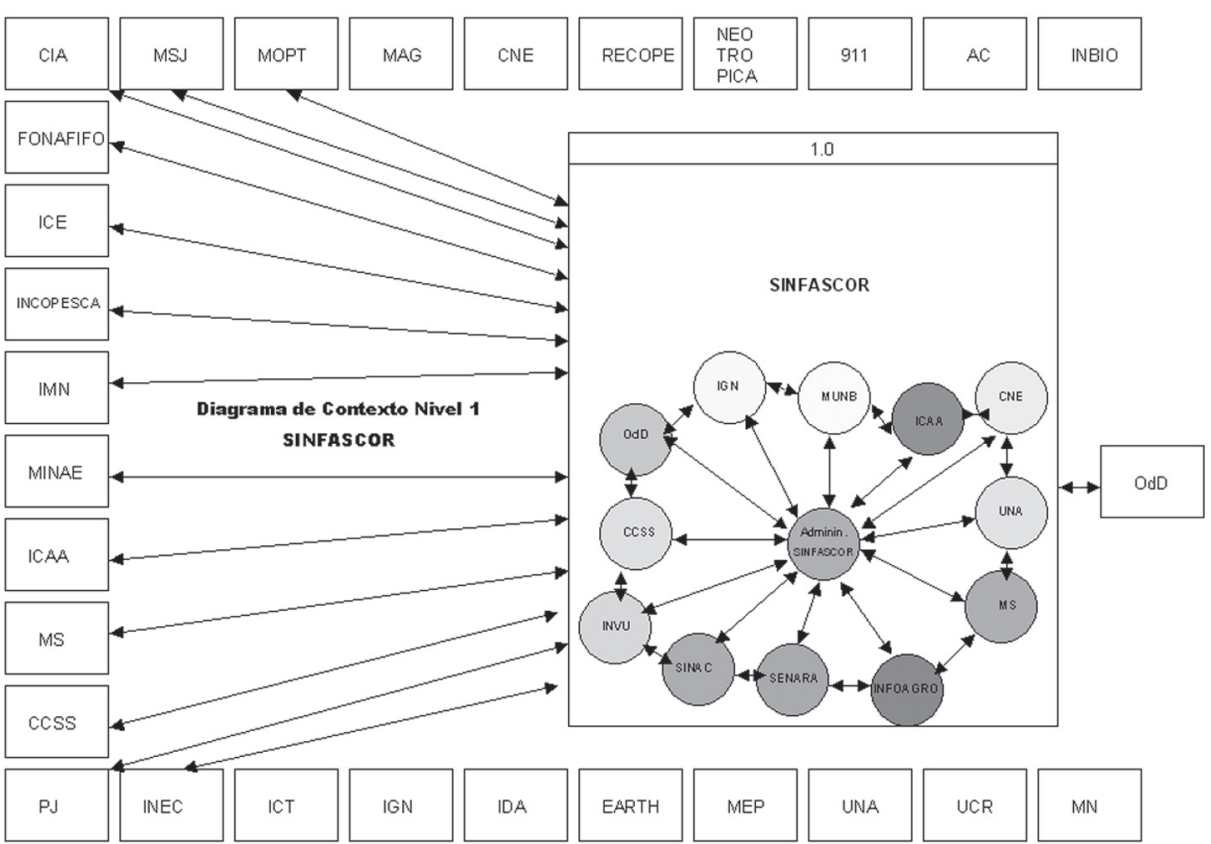

Figura A.4 


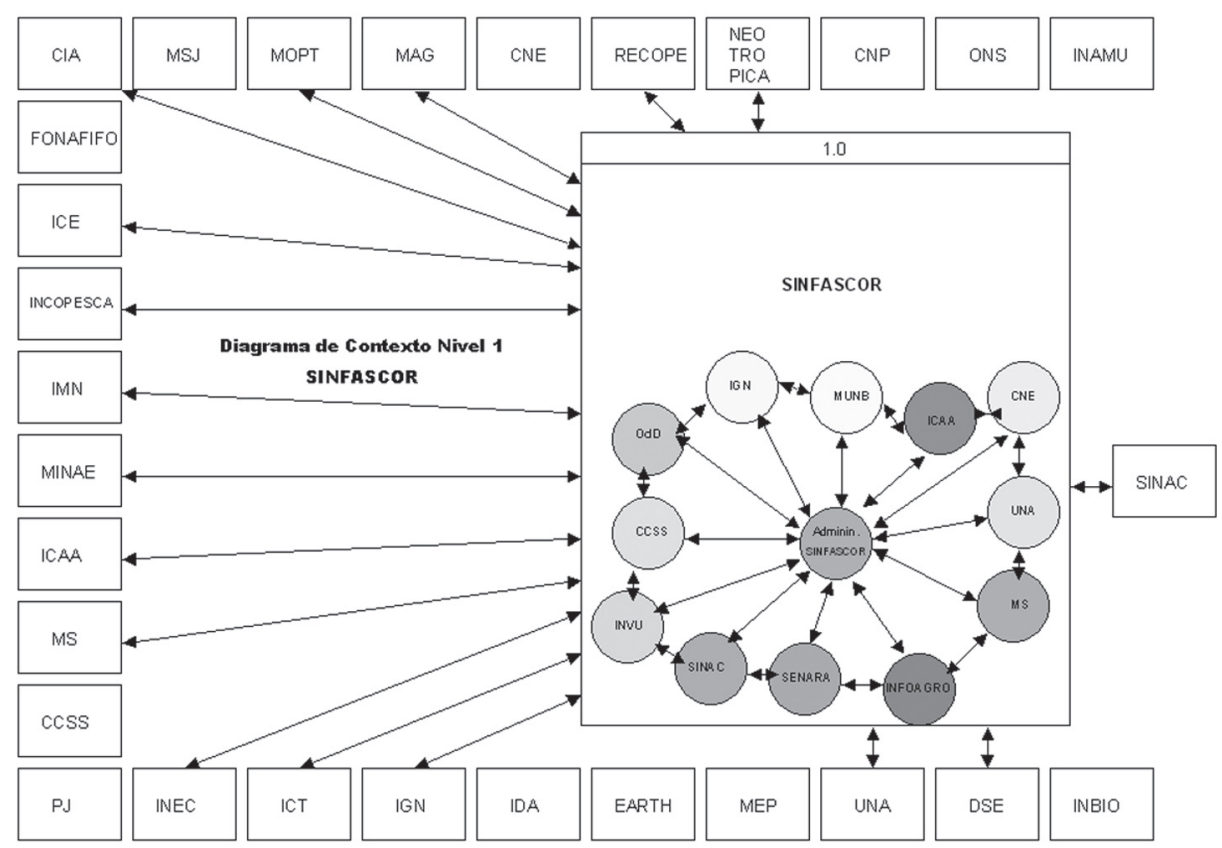

Figura A.5

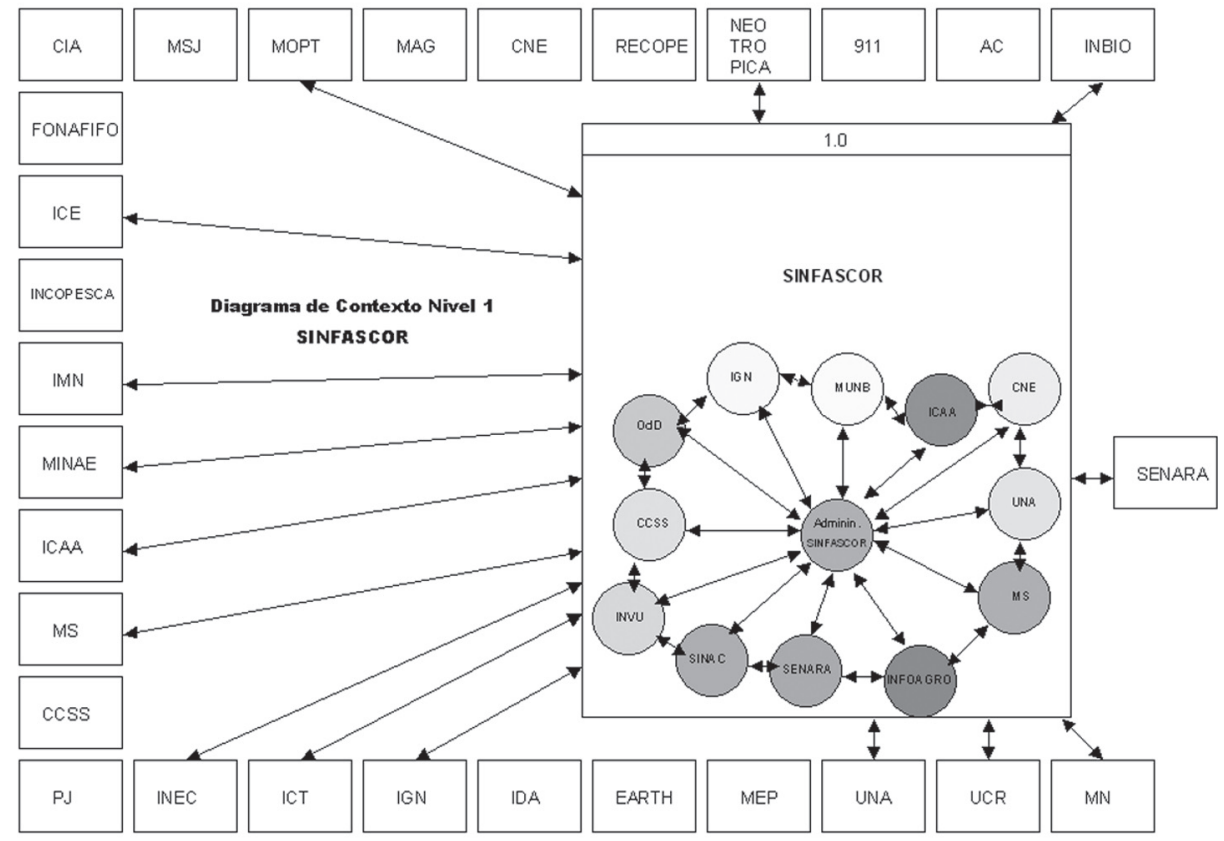

Figura A.6 


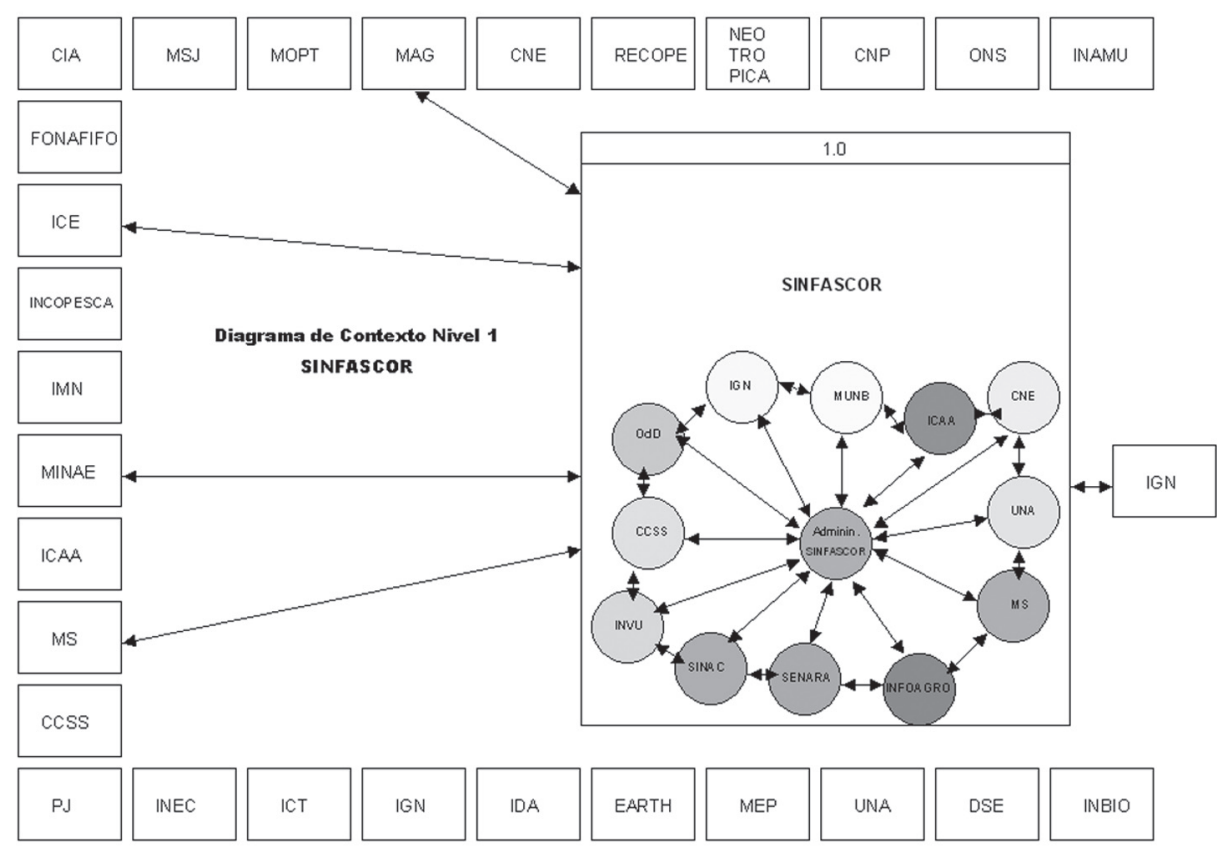

Figura A.7

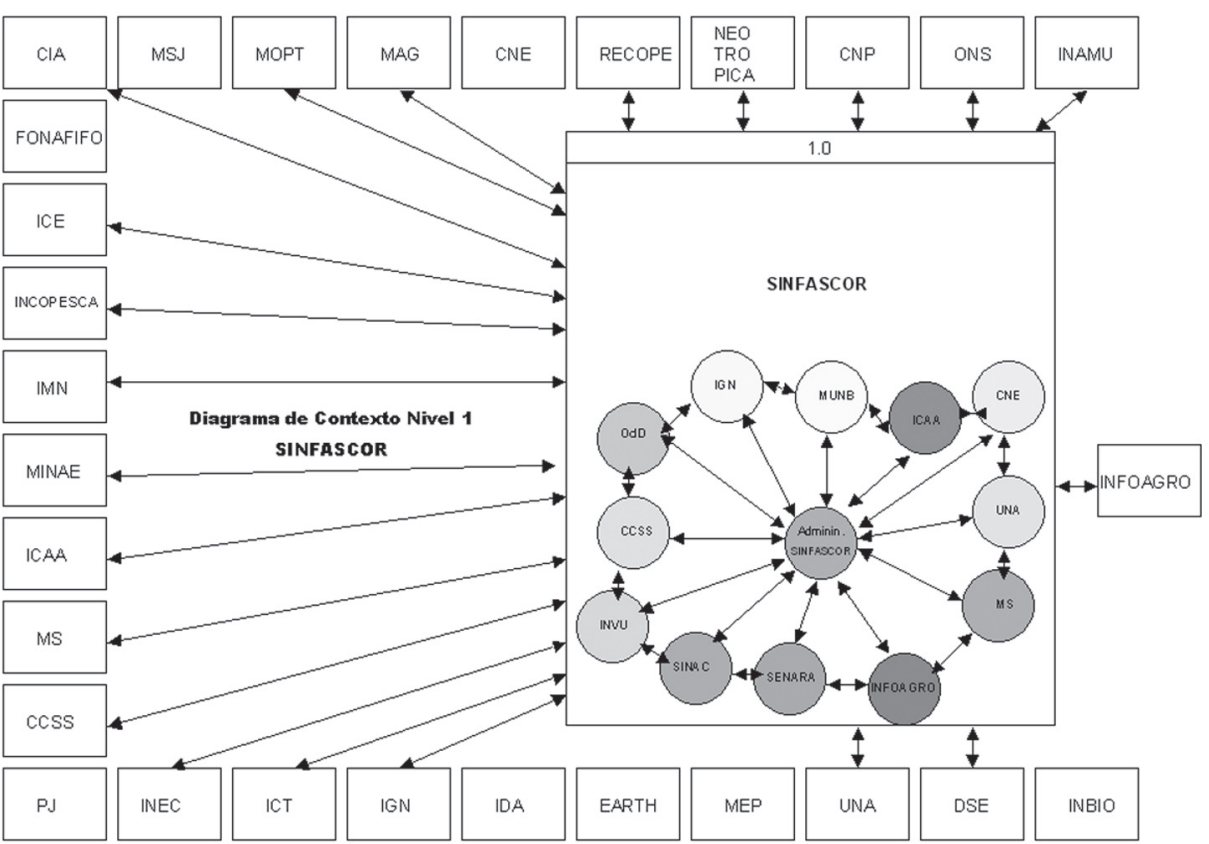

Figura A.8 


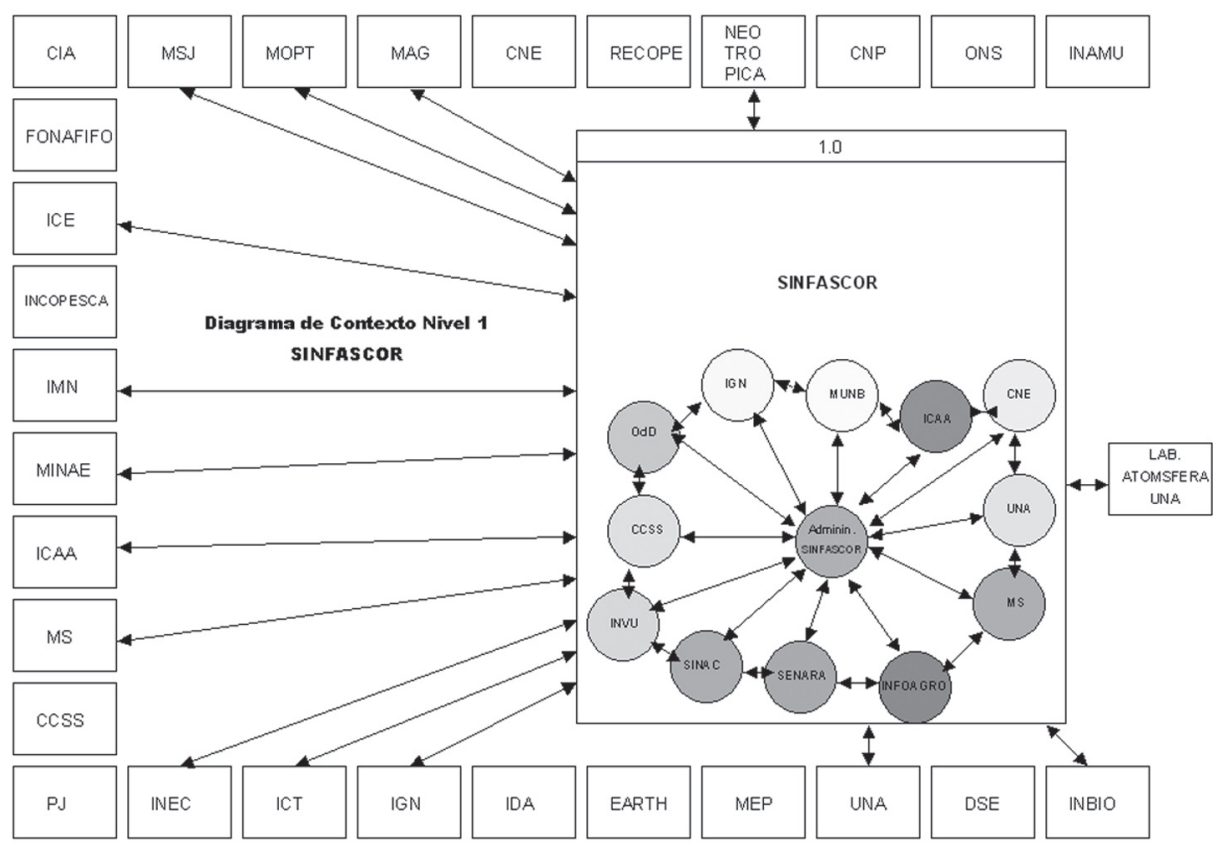

Figura A.9

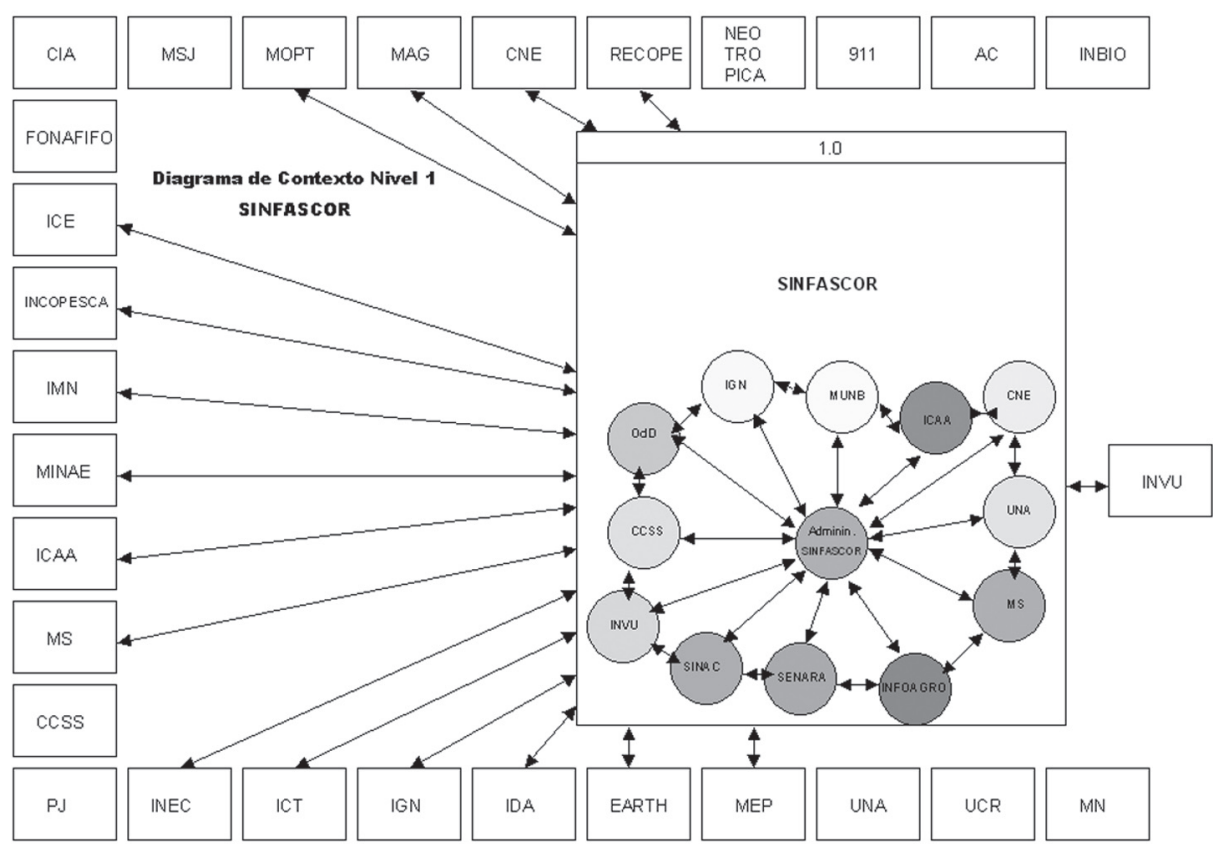

Figura A.10 


\section{BIBLIOGRAFÍA}

Cruz, N. (1996, Noviembre). Secuencia metodológica para el desarrollo de proyectos del abastecimiento de agua potable.

Coronado, O. (2001). Crítica a la Teoría General de Sistemas: la Teoría de Redes como un complemento ilustrada con el diseño de un Sistema Nacional de Información en Ambiente y Salud. Revista Ingeniería, 11 (1 y 2), 69-79.

\section{Entrevistas}

Entrevista realizada al Arq. Luis Fernando Acuña de la Unidad de Planificación Territorial del Instituto Nacional de Vivienda y Urbanismo (INVU) el día 12 de julio del 2000.

Entrevista realizada al Arq. William Rivera, Analista de Recursos Naturales del Instituto Nacional de Vivienda y Urbanismo (INVU el día 12 de julio del 2000.

Entrevista realizada al Dr. Edgar Gutiérrez Ezpeleta, Director del Observatorio del Desarrollo de la Universidad de Costa Rica (OdD) el día 11 de julio del 2000.

Entrevista realizada al Ing. Edwin Matarrita, Jefe del Sistema de Información Geográfico del Instituto Costarricense de Acueductos y Alcantarillados (ICAA) el día 27 de julio del 2000.

Entrevista realizada el Ing. Federico Arellano, Jefe del Departamento de Aguas Subterráneas del Instituto Costarricense de Acueductos y Alcantarillados (ICAA) el día 1 de agosto del 2000.

Entrevista realizada el Ing. José Luis Arguedas Negrini, Director del Departamento de Estudioy Proyectos delInstitutoCostarricense de Acueductos y Alcantarillados (ICAA) el día 1 de agosto del 2000.

Entrevista realizada al Lic. Gerardo Ramírez, Jefe del Departamento de Cuencas del
Instituto Costarricense de Acueductos y Alcantarillados (ICAA) el día 1 de agosto del 2000.

Entrevista realizada a la Licda. Lorena Echandi, Jefa del Departamento de Informática del Instituto Costarricense de Acueductos y Alcantarillados (ICAA) el día 20 del julio del 2000.

Entrevista realizada a la Licda. Marjorie Mora Valverde del Observatorio del Desarrollo de la Universidad de Costa Rica (OdD) los días 18, 19, 20 de julio del 2000.

Entrevista realizada al Sr. Sergio Sánchez de la Comisión Nacional de Emergencias los días 21 y 28 de julio del 2000.

Entrevista realizada al Sr. Walter Ramírez, Jefe del Departamento d Estudios Básicos del Instituto Costarricense de Acueductos y Alcantarillados (ICAA) el día 28 de julio del 2000.

\section{Medios electrónicos en Internet.}

http://www.cne.go.cr

http://www.odd.ucr.ac.cr

http://www.rds.ucr.ac.cr

\section{Medios Audiovisuales}

Observatorio del Desarrollo. (1999). Tendencias del Desarrollo Costarricense. Series Cronológicas 1985 - 1998.(CD-ROM) (San José)

\section{SOBRE EL AUTOR}

\section{Oscar Coronado Jurado}

Candidato a doctor.

Subdirector, Escuela de Ingeniería Industrial, Universidad de Costa Rica.

Teléfonos: 207-4085, Facsímil. 272-5196

Correo electrónico: oscorona@racsa.co.cr 
